\title{
Improvement of the Simulation of Cloud Longwave Scattering in Broadband Radiative Transfer Models
}

\author{
Guanglin Tang, ${ }^{\mathrm{a}}$ Ping Yang, ${ }^{\mathrm{a}}$ GeOrge W. Kattawar, ${ }^{\mathrm{b}, \mathrm{c}}$ Xianglei Huang, ${ }^{\mathrm{d}}$ Eli J. Mlawer, \\ BRYAN A. BAUM, ${ }^{\mathrm{f}}$ AND MICHAEL D. KING ${ }^{\mathrm{g}, \mathrm{a}}$ \\ ${ }^{a}$ Department of Atmospheric Sciences, Texas A\&M University, College Station, Texas \\ ${ }^{\mathrm{b}}$ Department of Physics and Astronomy, Texas A\&M University, College Station, Texas \\ ${ }^{\mathrm{c}}$ Institute for Quantum Science and Engineering, Texas A\&M University, College Station, Texas \\ ${ }^{\mathrm{d}}$ Department of Climate and Space Sciences and Engineering, University of Michigan, Ann Arbor, Michigan \\ ${ }^{\mathrm{e}}$ Atmospheric and Environmental Research, Inc., Cambridge, Massachusetts \\ ${ }^{\mathrm{f}}$ Space Science and Engineering Center, University of Wisconsin-Madison, Madison, Wisconsin \\ ${ }^{\mathrm{g}}$ Laboratory for Atmospheric and Space Physics, University of Colorado Boulder, Boulder, Colorado
}

(Manuscript received 11 January 2018, in final form 25 March 2018)

\begin{abstract}
Cloud longwave scattering is generally neglected in general circulation models (GCMs), but it plays a significant and highly uncertain role in the atmospheric energy budget as demonstrated in recent studies. To reduce the errors caused by neglecting cloud longwave scattering, two new radiance adjustment methods are developed that retain the computational efficiency of broadband radiative transfer simulations. In particular, two existing scaling methods and the two new adjustment methods are implemented in the Rapid Radiative Transfer Model (RRTM). The results are then compared with those based on the Discrete Ordinate Radiative Transfer model (DISORT) that explicitly accounts for multiple scattering by clouds. The two scaling methods are shown to improve the accuracy of radiative transfer simulations for optically thin clouds but not effectively for optically thick clouds. However, the adjustment methods reduce computational errors over a wide range, from optically thin to thick clouds. With the adjustment methods, the errors resulting from neglecting cloud longwave scattering are reduced to less than $2 \mathrm{~W} \mathrm{~m}^{-2}$ for the upward irradiance at the top of the atmosphere and less than $0.5 \mathrm{~W} \mathrm{~m}^{-2}$ for the surface downward irradiance. The adjustment schemes prove to be more accurate and efficient than a four-stream approximation that explicitly accounts for multiple scattering. The neglect of cloud longwave scattering results in an underestimate of the surface downward irradiance (cooling effect), but the errors are almost eliminated by the adjustment methods (warming effect).
\end{abstract}

\section{Introduction}

It is commonly assumed that cloud longwave scattering is unimportant for estimating the atmospheric energy budget and thus is neglected in general circulation model (GCM) irradiance simulations and in radiative transfer simulations for deriving the radiation budgets from retrieved cloud optical properties. A number of studies indicate a range of overestimates of top-of-theatmosphere (TOA) longwave irradiance resulting from neglecting longwave scattering. For example, Stephens et al. (2001) showed an overestimation of global-average outgoing longwave radiation (OLR) of approximately $8 \mathrm{~W} \mathrm{~m}^{-2}$ when cloud longwave scattering is neglected in a GCM, whereas regional overestimation could be as

\footnotetext{
Corresponding author: Guanglin Tang, tangguanglin@gmail.com
}

large as $20 \mathrm{Wm}^{-2}$. Costa and Shine (2006) showed a global overestimation of OLR of approximately $3 \mathrm{~W} \mathrm{~m}^{-2}$ by neglecting cloud longwave scattering, with low-level clouds contributing $0.9 \mathrm{~W} \mathrm{~m}^{-2}$, midlevel clouds contributing $0.7 \mathrm{~W} \mathrm{~m}^{-2}$, and high clouds contributing $1.4 \mathrm{~W} \mathrm{~m}^{-2}$. Other studies also reported OLR overestimations resulting from neglecting cloud longwave scattering with values ranging from 1.5 to $11 \mathrm{~W} \mathrm{~m}^{-2}$ (Ritter and Geleyn 1992; Joseph and Min 2003; Schmidt et al. 2006). In a recent sensitivity study, Kuo et al. (2017) showed that neglecting cloud longwave scattering could result in an overestimation of the global-average OLR of approximately $2.6 \mathrm{~W} \mathrm{~m}^{-2}$ and an overestimation of the surface net upward radiation of approximately $1.1 \mathrm{~W} \mathrm{~m}^{-2}$.

Such significant changes caused by excluding cloud longwave scattering effects suggest a pressing need for 
more accurate longwave radiative transfer (RT) simulations in the broadband radiance models used in GCMs. However, such a change should not substantially increase the computational burden, since the computational costs of the RT models in GCMs are already high. One approach for mitigating the computational burden is to scale the cloud absorption optical thickness, as in the scheme developed by Chou et al. (1999). The Chou et al. scheme retains the computational efficiency by neglecting the longwave scattering and improves accuracy for irradiance in the case of optically thin clouds. However, there is little improvement for optically thick clouds, as the TOA longwave irradiance is still overestimated by approximately $4 \mathrm{Wm}^{-2}$. Therefore, simply scaling the cloud optical thickness in conjunction with no-scattering simulations is insufficient for further improvement of irradiance calculations over a range of optical thicknesses.

The similarity principle for radiative transfer (van de Hulst 1974; Sobolev 1975; Joseph et al. 1976; van de Hulst 1980; Twomey and Bohren 1980; McKellar and Box 1981; Ding et al. 2017) provides an alternative approach for scaling the optical thickness. The basic concept is that radiance or irradiance simulations may have similar results with two different sets of cloud optical properties if the optical properties satisfy certain relationships. With this principle, one could consider replacing a scattering cloud layer with a nearly nonscattering cloud layer and obtain similar irradiance values. The similarity principle has been verified for TOA solar radiance calculations (with an external source and a nonemitting atmosphere) with a singlescattering albedo $\omega$ between 0.8 and 1 and an asymmetry factor $g$ between 0.75 and 0.9 (Ding et al. 2017). However, further investigation is necessary for the case of small $\omega$ (i.e., strongly absorptive case) and for longwave irradiance from a scattering-emitting cloud layer where there is no external source term. As with the Chou et al. scheme, a simple scaling strategy also lacks the ability to provide accurate irradiance calculations for optically thick clouds. These two schemes are compared in this study (section 3) and have similar results for the irradiance simulations.

The primary goal of this study is to develop a new adjustment scheme based on the similarity principle and the Chou scaling schemes. We will show that this adjustment scheme improves the longwave irradiance calculations in a broadband radiative transfer model for both optically thin and thick ice clouds. Although the present scheme also works for low-level liquid water clouds, we will not show results for this case because the scattering effect is generally weak in the longwave regime.

The remaining part of the paper is organized as follows: Section 2 discusses the methodology. Section 3 presents the simulation results and discussions. Section 4 summarizes and concludes the study.

\section{Methodology}

\section{a. Similarity principle}

Previous derivations of the similarity relation focused on a nonemitting atmosphere (McKellar and Box 1981; van de Hulst 1974; King 1981; Ding et al. 2017) that can be extended to an emitting atmosphere straightforwardly following the derivation of Ding et al. (2017). For longwave scattering without external sources, the radiance is independent of azimuth. The azimuthally averaged radiative transfer equation (RTE) for a plane-parallel atmosphere, following Liou [1980, Eq. (6.1.12)], is

$$
\begin{aligned}
\mu \frac{\partial I(\tau, \mu)}{\partial \tau}= & I(\tau, \mu)-[1-\omega(\tau)] B(\tau) \\
& -\frac{\omega(\tau)}{2} \int_{-1}^{1} I\left(\tau, \mu^{\prime}\right) P\left(\mu, \mu^{\prime}\right) d \mu^{\prime},
\end{aligned}
$$

where $I$ is the radiance, $\mu$ and $\mu^{\prime}$ indicate the cosines of the angles between the propagating direction of $I$ and the zenith (positive for upward radiance and negative for downward radiance), $\tau$ is the optical thickness (zero at TOA and positive at the surface), $B$ is the blackbody radiance (Planck function of temperature and wavelength), and $P\left(\mu, \mu^{\prime}\right)$ is the azimuthally averaged scattering phase function. The dependence of Eq. (1) on wavelength is implied. By multiplying Eq. (1) with the $l$ th-order Legendre polynomial $p_{l}(\mu)$, followed by integrating over $\mu$, the result is

$$
\int_{-1}^{1} \mu \frac{\partial I(\tau, \mu)}{\partial \tau} p_{l}(\mu) d \mu=\int_{-1}^{1} I(\tau, \mu) p_{l}(\mu) d \mu-[1-\omega(\tau)] B(\tau) \delta_{0 l}-\frac{\omega(\tau)}{2} \int_{-1}^{1} \int_{-1}^{1} I\left(\tau, \mu^{\prime}\right) P\left(\mu, \mu^{\prime}\right) p_{l}(\mu) d \mu^{\prime} d \mu
$$

where $\delta_{0 l}$ is the Kronecker delta function. Expansion of $P\left(\mu, \mu^{\prime}\right)$ in terms of Legendre polynomials and application of the addition theorem (Edmonds 2016; Wiscombe 1977) yields 


$$
P\left(\mu, \mu^{\prime}\right)=\sum_{l=0}^{\infty}(2 l+1) \chi_{l} p_{l}(\mu) p_{l}\left(\mu^{\prime}\right),
$$

where $\chi_{l}$ is the $l$ th coefficient of the Legendre expansion of the phase function. By noting the orthogonality of Legendre polynomials, we substitute Eq. (3) into Eq. (2) to obtain

$$
\begin{aligned}
& \int_{-1}^{1}\left\{\mu \frac{\partial I(\tau, \mu)}{\partial \tau}-I(\tau, \mu)\left[1-\omega(\tau) \chi_{l}\right]\right\} p_{l}(\mu) d \mu \\
& \quad=-[1-\omega(\tau)] B(\tau) \delta_{0 l} .
\end{aligned}
$$

From Eq. (4), obtaining an invariant $I(\tau, \mu)$ with a different set of optical properties requires that

$$
\tau^{\prime}\left(1-\omega^{\prime} \chi_{l}^{\prime}\right)=\tau\left(1-\omega \chi_{l}\right), \quad l=0,1,2, \ldots \infty,
$$

which is true because the thermal emission term vanishes for all $l$ other than zero. Variables with or without primes indicate two different sets of optical properties. This similarity relation is the same as for a nonemitting atmosphere (McKellar and Box 1981). Normally, it is impossible to satisfy Eq. (5) for all $l$ except when two sets of optical properties are exactly the same. However, we may consider the first two lowest-order values for $l$ to obtain approximate similarity relations. Specifically, $l=0$ and $l=1\left(\chi_{0}=1\right.$ and $\left.\chi_{1}=g\right)$ result in the following two similarity relations, respectively, which involve three important variables, $\tau, \omega$, and $g$ :

$$
\begin{aligned}
\tau^{\prime}\left(1-\omega^{\prime}\right) & =\tau(1-\omega), \\
\tau^{\prime}\left(1-\omega^{\prime} g^{\prime}\right) & =\tau(1-\omega g) .
\end{aligned}
$$

If $\tau^{\prime}, \omega^{\prime}$, and $g^{\prime}$ are considered as unknowns, then Eq. (6) has 1 degree of freedom. Setting $g^{\prime}=0$ (isotropic scattering) results in

$$
\tau^{\prime}=\tau(1-\omega g), \quad \omega^{\prime}=\frac{\omega(1-g)}{(1-\omega g)} .
$$

For an isotropic scattering layer that has been used to perform the two-stream approximation for the longwave radiative transfer in the GCM Model I (Hansen 1969; Hansen et al. 1983), this is relatively accurate for a single layer but the accuracy degrades for multiple layers combined using the adding method.

In contrast, keeping only the first-order $l=0$ results in Eq. (6a), which still has a single degree of freedom. Setting $\omega^{\prime}=0$ yields

$$
\tau^{\prime}=\tau(1-\omega), \quad \omega^{\prime}=0,
$$

which implies no scattering. In other words, neglecting the scattering with $\tau^{\prime}=\tau(1-\omega)$ corresponds to assuming that all scattering is solely in the forward direction (i.e., $g=1$ ), which can be interpreted as direct transmission. The reason the TOA irradiance is overestimated is because the side- and backscattering of the upward radiance is accounted for as being directly transmitted to TOA.

To mitigate the aforesaid flaw, one could consider scaling (increasing) the absorption optical thickness to compensate for the overestimated upward transmission. However, with $\omega^{\prime}=0$, Eq. (6) has no solution. We choose a $\tau^{\prime}$ value so that the square error of Eq. (6) is minimized under the condition of $\omega^{\prime}=0$ :

$$
\tau^{\prime}=\tau\left(1-\omega \frac{1+g}{2}\right), \quad \omega^{\prime}=0
$$

The difference between this scaled optical thickness $\tau^{\prime}=\tau[1-\omega(1+g) / 2]$ and the absorbing optical thickness $\tau^{\prime}=\tau(1-\omega)$ is the term $\tau \omega(1-g) / 2$, which is half of what is identified as the scattering optical thickness according to Twomey and Bohren (1980). Qualitatively, because $\tau[1-\omega(1+g) / 2]$ is larger than $\tau(1-\omega)$, we expect a reduction of the overestimation of TOA irradiance. However, such a simple optical thickness scaling may not be sufficient for optically thick clouds. Thus, multiple scattering needs to be treated in a more rigorous way.

\section{b. Chou's parameterization for adjusting the cloud optical thickness}

In addition to the similarity principle scaling scheme, another scheme for adjusting the cloud optical thickness was developed by Chou et al. (1999) and is briefly summarized as follows. We approximate the upward and downward radiance with two respective constants. For example, the Chou et al. (1999) approach approximates the radiance in the integration term in Eq. (1) in the form

$$
I\left(\tau, \mu^{\prime}\right) \approx\left\{\begin{array}{cc}
I(\tau, \mu), & \mu \times \mu^{\prime}>0 \\
B(\tau), & \mu \times \mu^{\prime}<0
\end{array} .\right.
$$

In this way, the ambient radiance $I\left(\tau, \mu^{\prime}\right)$ in the integral term in the RTE Eq. (1) is assumed to be isotropic in each of the upward and downward hemispheres. It equals the incident radiance $I(\tau, \mu)$ when they are in the same hemisphere and equals the ambient blackbody radiance when they are in different hemispheres. For example, when solving the downward radiance, all downward radiance terms in Eq. (1) are assumed to be a single unknown, while all upward radiance terms are approximated in terms of the Planck radiation. Hence, the downward and upward radiances are decoupled in Eq. (1). The integration source term in Eq. (1) can be expressed as 


$$
\int_{-1}^{1} I\left(\tau, \mu^{\prime}\right) P\left(\mu, \mu^{\prime}\right) d \mu^{\prime} \approx\left\{\begin{array}{l}
I(\tau, \mu) \int_{-1}^{0} P\left(\mu, \mu^{\prime}\right) d \mu^{\prime}+B(\tau) \int_{0}^{1} P\left(\mu, \mu^{\prime}\right) d \mu^{\prime}, \quad \mu<0 \\
I(\tau, \mu) \int_{0}^{1} P\left(\mu, \mu^{\prime}\right) d \mu^{\prime}+B(\tau) \int_{-1}^{0} P\left(\mu, \mu^{\prime}\right) d \mu^{\prime}, \quad \mu>0
\end{array}\right.
$$

The terms $\int_{0}^{1} P\left(\mu, \mu^{\prime}\right) d \mu^{\prime}$ and $\int_{-1}^{0} P\left(\mu, \mu^{\prime}\right) d \mu^{\prime}$ are functions of $\mu$, but they can be approximated by their averages over $\mu$ so as to be constants; note that the sum of these two terms is 2 because they are normalized. Then Eq. (11) is rewritten as

$$
\int_{-1}^{1} I\left(\tau, \mu^{\prime}\right) P\left(\mu, \mu^{\prime}\right) d \mu^{\prime} \approx \begin{cases}I(\tau, \mu) \times(2-2 b)+B(\tau) \times 2 b, & \mu<0 \\ I(\tau, \mu) \times(2-2 b)+B(\tau) \times 2 b, & \mu>0\end{cases}
$$

where

$$
\begin{aligned}
b \equiv & \frac{1}{2} \int_{0}^{1} d \mu \int_{-1}^{0} P\left(\mu, \mu^{\prime}\right) d \mu^{\prime} \equiv \frac{1}{2} \int_{-1}^{0} d \mu \int_{0}^{1} P\left(\mu, \mu^{\prime}\right) d \mu^{\prime} \\
& \approx 1-\sum_{i=1}^{4} a_{i} g^{i-1}
\end{aligned}
$$

with $a_{1}=0.5, a_{2}=0.3738, a_{3}=0.0076$, and $a_{4}=0.1186$ (Chou et al. 1999). Note that the phase function $P\left(\mu, \mu^{\prime}\right)$ is approximated in terms of the Henyey-Greenstein function - that is, a function of only $g, \mu$, and $\mu^{\prime}$-and is fitted with a fourth-order polynomial. Substituting Eq. (12) into Eq. (1) yields

$$
\mu \frac{\partial I(\tau, \mu)}{[1-\omega(1-b)] \partial \tau}=I(\tau, \mu)-B(\tau) .
$$

Because $\omega$ is assumed to be constant in each layer in radiative transfer models (RTMs), the role of the factor $1-\omega(1-b)$ in Eq. (14) is simply to scale the optical thickness and to represent the radiative transfer equation in a nonscattering form.

With the similarity principle approach, replacing $1-b$ with $(1+g) / 2$ in Eq. (14) leads to a similar radiative transfer equation:

$$
\mu \frac{\partial I(\tau, \mu)}{\left[1-\omega \frac{(1+g)}{2}\right] \partial \tau}=I(\tau, \mu)-B(\tau)
$$

The only difference between the similarity principle scaling scheme and Chou's scaling scheme is the scaling factor $1-\omega(1+g) / 2$ versus $[1-\omega(1-b)]$. Note that when $g=1$ or $\omega=0$, both schemes reduce to the original scheme that neglects scattering:

$$
\mu \frac{\partial I(\tau, \mu)}{(1-\omega) \partial \tau}=I(\tau, \mu)-B(\tau)
$$

\section{c. Adjustment scheme}

In the Chou et al. (1999) scaling scheme, the major approximation is with the hemispheric isotropic radiance. The performance of the scheme highly depends on the extent to which this approximation holds true. While one can consider the ambient radiance as being approximately equal to the incident radiance in the same hemisphere, large biases may result from this approximation when the incident radiance is in the opposite hemisphere, especially for downward ambient radiance on top of a cloud layer. In this case, the downward ambient radiance is much weaker than the blackbody radiance. Hence, we reconsider the approximation for the downward ambient radiance when solving for the upward radiance.

When neglecting scattering, RTMs first solve for the downward radiance, layer by layer from the TOA to the surface, and then the upward radiance from the surface to the TOA. The governing equation [Eq. (16)] is a firstorder ordinary differential equation with a boundary condition that the downward radiance at TOA is zero. In such a process, when solving for the upward radiance, the downward radiance is already known. This means that the ambient downward radiance is not necessarily assumed to be the blackbody radiance when solving for the upward radiance. Equation (12) then becomes

$$
\int_{-1}^{1} I\left(\tau, \mu^{\prime}\right) P\left(\mu, \mu^{\prime}\right) d \mu^{\prime} \approx\left\{\begin{array}{ll}
I(\tau, \mu) \times(2-2 b)+B(\tau) \times 2 b, & \mu<0 \\
I(\tau, \mu) \times(2-2 b)+I(\tau,-\mu) \times 2 b, & \mu>0
\end{array} .\right.
$$


Note that the term $I(\tau,-\mu)$ in the upward direction $(\mu>0)$ is obtained by solving for the downward

direction first. Substitution of Eq. (17) into Eq. (1) yields

$$
\begin{array}{ll}
\mu \frac{\partial I(\tau, \mu)}{[1-\omega(1-b)] \partial \tau}=I(\tau, \mu)-B(\tau), & \mu<0 . \\
\mu \frac{\partial I(\tau, \mu)}{[1-\omega(1-b)] \partial \tau}=I(\tau, \mu)-B(\tau)-\frac{\omega b}{1-\omega(1-b)}[I(\tau,-\mu)-B(\tau)], & \mu>0 .
\end{array}
$$

Note the additional term $-\omega b /[1-\omega(1-b)] \times[I(\tau,-\mu)-$ $B(\tau)]$ in Eq. (18b), which is the only difference between the new scheme and the Chou et al. scheme. We use Eq. (18a) to solve for the downward radiance from the TOA to the surface, and then substitute the resulting downward radiance into Eq. (18b) to solve for the upward radiance from the surface to the TOA. The process proceeds layer by layer. A specific solution is necessary for solving the upward radiance at the $(n+1)$ th level from the $n$th level, which satisfies

$$
\mu \frac{\partial I^{\mathrm{ss}}(\tau, \mu)}{[1-\omega(1-b)] \partial \tau}=I^{\mathrm{ss}}(\tau, \mu)-\frac{\omega b}{1-\omega(1-b)}[I(\tau,-\mu)-B(\tau)], \quad \mu>0
$$

with a boundary condition of $I^{\mathrm{ss}}\left(\tau_{n}, \mu\right)=0$. Here $I^{\mathrm{ss}}$ denotes the specific solution for the $n$th layer $\tau \in\left[\tau_{n+1}, \tau_{n}\right]$, and $\tau_{n}, n=0,1, \ldots, N$ is the optical depth at the top of the $n$th layer ( $n=0$ is for the surface and $n=N$ is for TOA).
For simplicity, we assume $B$ to be constant within a layer, which is reasonable when the temperature varies little within a layer. Then, from Eq. (18a) we know that $I(\tau,-\mu)-B(\tau)$ changes exponentially with $\tau$ and is given by

$$
I(\tau,-\mu)-B(\tau)=\left[I\left(\tau_{n+1},-\mu\right)-B\left(\tau_{n+1}\right)\right] \exp \left\{-\frac{[1-\omega(1-b)]\left(\tau-\tau_{n+1}\right)}{\mu}\right\}, \quad \text { for } \quad \mu>0, \tau_{n+1}<\tau<\tau_{n}
$$

Note that $B$ is assumed constant in this process. Substitution of Eq. (20) into Eq. (19) results in a firstorder ordinary differential equation involving $I^{\mathrm{ss}}(\tau, \mu)$.
Furthermore, $I\left(\tau_{n+1},-\mu\right)$ is known from the previous process for solving for the downward radiances. The solution for the differential equation leads to

$$
\begin{aligned}
I^{\mathrm{ss}}\left(\tau_{n+1}, \mu\right)= & \frac{\omega b}{2[1-\omega(1-b)]}\left(\left[I\left(\tau_{n+1},-\mu\right)-B\left(\tau_{n+1}\right)\right]-\left[I\left(\tau_{n},-\mu\right)-B\left(\tau_{n}\right)\right]\right. \\
& \left.\times \exp \left\{-\frac{[1-\omega(1-b)]\left(\tau_{n}-\tau_{n+1}\right)}{\mu}\right\}\right), \quad \text { for } \quad \mu>0,
\end{aligned}
$$

where the exponential term $\exp \{-[1-\omega(1-b)]$ $\left.\left(\tau_{n}-\tau_{n+1}\right) / \mu\right\}$ is the transmissivity of the $n$th layer after scaling. We call this specific solution the adjustment term, and it is determined from the known downward radiance. To be more accurate, $B$ can be assumed to be a linear function of $\tau$, for example, in the RRTM (Mlawer et al. 1997; Iacono et al. 2000). However, as long as the temperature does not change significantly within a layer, the exponential solution for the downward radiance given in Eq. (20) is still a reasonable approximation. Hence, the final solution of the upward radiance at the $(n+1)$ th level is the general solution to the nonscattering radiative equation [Eq. (14)] plus the specific upward radiance adjustment given by Eq. (21). We call the scheme with this solution the Chou scaling-adjustment scheme. The specific solution is basically negative, leading to a 
reduction of the overestimates of the upward irradiance, especially above high clouds. In particular, when the layer is very thin, in Eq. (21) $I\left(\tau_{n+1},-\mu\right)-B\left(\tau_{n+1}\right)$ is similar to $I\left(\tau_{n},-\mu\right)-B\left(\tau_{n}\right)$ and the transmissivity term $\exp \left\{-[1-\omega(1-b)]\left(\tau_{n}-\tau_{n+1}\right) / \mu\right\}$ approaches unity and therefore the adjustment term is very small. When the layer is optically thick and high, $\exp \left\{-[1-\omega(1-b)]\left(\tau_{n}-\right.\right.$ $\left.\left.\tau_{n+1}\right) / \mu\right\}$ and $I\left(\tau_{n+1},-\mu\right)$ approach zero and therefore the adjustment term approaches $-\omega b / 2[1-\omega(1-b)] \times$ $B\left(\tau_{n+1}\right)$, a negative value. Also, the adjustment increases with cloud optical thickness, which is ideal for mitigating the weakness of the two scaling schemes for optically thick clouds.

Alternatively, the term $(1-b)$ may be replaced with $(1+g) / 2$ in Eqs. (14) and (21) to obtain general and specific solutions based on the similarity principle. We call the scheme with this solution the similarity scaling-adjustment scheme. In Chou's scheme, $(1-b)$ represents the percentage of the scattering contribution in the direction of the to-be-solved radiance from the ambient radiance in the same hemisphere, and $b$ represents the contribution from the ambient radiance in the opposite hemisphere. In the similarity scaling-adjustment scheme, with $(1+g) / 2$ replacing $(1-b)$, the percentage contributions from the ambient radiance in the two hemispheres are $(1+g) / 2$ and $(1-g) / 2$, respectively. When $g=1$, the scattering contribution to the to-be-solved radiance is purely from the ambient radiance in the same hemisphere. When $g=$ 0 , the contributions from the two hemispheres are equal, corresponding to isotropic scattering. The appendix describes the physical meaning of the similarity principle scaling and the adjustment term. In a simplified model where the atmosphere is a one-dimensional, optically thin layer, the similarity principle scaling scheme results in more accurate TOA irradiance than the neglect-scattering scheme, and the similarity scaling-adjustment scheme results in the exact solution, completely offsetting the error of the scaling scheme.

After solving for the upward radiance, the downward radiance can be adjusted by using the known upward radiance following an equation similar to Eq. (21) but from the TOA to the surface:

$$
\begin{aligned}
I^{\mathrm{ss}}\left(\tau_{n-1}, \mu\right)= & \frac{\omega b}{2[1-\omega(1-b)]}\left(\left[I\left(\tau_{n-1},-\mu\right)-B\left(\tau_{n-1}\right)\right]-\left[I\left(\tau_{n},-\mu\right)-B\left(\tau_{n}\right)\right]\right. \\
& \left.\times \exp \left\{-\frac{[1-\omega(1-b)]\left(\tau_{n-1}-\tau_{n}\right)}{-\mu}\right\}\right), \text { for } \mu<0,
\end{aligned}
$$

where $n=1,2, \ldots, N$ is the $n$th level, and then the solved downward radiance can change the upward radiance solution again. This iterative process may lead to a considerable increase of computational time. Alternatively, we can optimize the scale of the adjustment term given by Eq. (21) to minimize the error for a number of simulations and use only "one and a half" iterative steps. Based on the simulations, the coefficient 0.5 (or $1 / 2$ ) in Eq. (21) can be replaced by values of 0.4 and 0.3 for the similarity and the Chou scaling-adjustment schemes, respectively. This coefficient optimization is partly because of several assumptions we have made. In summary, the first step of this approach is to solve for the downward radiance without adjustment and then to solve for the upward radiance with adjustment given by Eq. (21) with optimized coefficients. Eventually, the downward radiance is adjusted by the term given by Eq. (22) with the same optimized coefficient. The computational time is almost the same as the original scheme that neglects longwave scattering. All schemes used in this study along with their corresponding governing equations (and specific solution) and approximate computational time are listed in Table 1.

\section{Results}

We present results for the four schemes discussed in the previous section: the Chou et al. scaling approach, the similarity principle scaling approach, the Chou scalingadjustment scheme, and the similarity scaling-adjustment scheme. For evaluation purposes, we implement all four schemes in the RRTM longwave model, a simplified version [RRTM for General Circulation Models (RRTMG)] of which has been used to simulate radiative transfer in multiple GCMs, such as the Community Earth System Model (CESM; Kay et al. 2015) and the Weather Research and Forecasting (WRF) Model (Powers et al. 2017). In the RRTM longwave model, the upward and downward radiances are calculated for a set of radiance spectral bands, without considering scattering, for a single or multiple (1-4) angles, and then integrated to provide the irradiance. Cloud scattering can also be included using the DISORT option in RRTM, but it is much slower computationally.

For the purpose of generality, we use multiple ice cloud particle models to represent the ice cloud optical properties. In addition to the built-in spherical ice particle model (Hu and Stamnes 1993; Key 1996; 
TABLE 1. RRTM schemes and corresponding governing equations and computational times.

\begin{tabular}{|c|c|c|}
\hline Scheme & Governing equation & $\begin{array}{l}\text { Computational time (normalized to } \\
\text { original neglect-scattering scheme } \\
\text { with three angles) }\end{array}$ \\
\hline Original neglect-scattering scheme & Eq. (16) & 1 \\
\hline 16-stream DISORT & Eq. (1) & $\sim 100$ \\
\hline Similarity principle scaling scheme & Eq. (15) & $\sim 1$ \\
\hline Chou scaling scheme & Eq. (14) & $\sim 1$ \\
\hline $\begin{array}{l}\text { Adjustment scheme based on similarity } \\
\text { (or similarity scaling-adjustment scheme) }\end{array}$ & $\begin{array}{l}\text { Eqs. }(15),(21)^{\mathrm{a}} \text {, and }(22)^{\mathrm{a}} \text { with } 1-b \text { replaced } \\
\quad \text { by }(1+g) / 2\end{array}$ & $\sim 1$ \\
\hline $\begin{array}{l}\text { Adjustment scheme based on Chou (or } \\
\text { Chou scaling-adjustment scheme) }\end{array}$ & Eqs. $(14),(21)^{\mathrm{b}}$, and $(22)^{\mathrm{b}}$ & $\sim 1$ \\
\hline Four-stream approximation & Eq. (1) & $\sim 20$ \\
\hline
\end{tabular}

${ }^{\text {a }}$ Coefficient $1 / 2$ is replaced with 0.4 .

${ }^{\mathrm{b}}$ Coefficient $1 / 2$ is replaced with 0.3 .

denoted as the spherical model) and surface-roughened single-hexagonal-column model (Fu et al. 1998; denoted as the rough column model), we implement two more ice models, the surface-roughened eight-hexagonal-column aggregate model (Yang et al. 2013) used in the Moderate Resolution Imaging Spectroradiometer (MODIS) Collection 6 satellite cloud retrieval product (Platnick et al. 2017; denoted as the aggregate model) and the two-habit model consisting of a surface-roughened single-hexagonal-column and a 20-column aggregate model as a candidate for the Clouds and the Earth's Radiant Energy System (CERES)-MODIS Edition 5 product (Loeb et al. 2018; denoted as THM). In addition, six climatological atmospheric profiles are used: U.S. Standard Atmosphere, 1976 (COESA 1976); tropical atmosphere; midlatitude summer; midlatitude winter; high-latitude summer; and high-latitude winter. An ice cloud layer with a geometric thickness of $250 \mathrm{~m}$ is placed at high $(11 \mathrm{~km})$, middle $(8 \mathrm{~km})$, and lower altitudes $(5 \mathrm{~km})$. While clouds near the surface are generally liquid water phase, the assumption of ice phase for a cloud layer at $5 \mathrm{~km}$ and higher is reasonable for a sensitivity study. The cloud optical thickness varies from thin (0.1) to thick (10). The ice particle effective radius varies from 10 to $50 \mu \mathrm{m}$. Table 2 lists the detailed settings of atmosphere-cloud profiles used to evaluate the proposed schemes.

For each atmosphere-cloud profile, we use four newly implemented schemes along with the original neglectscattering scheme to calculate irradiances. Broadband radiances in one to four angles for both upward or downward directions are solved and integrated to obtain irradiances with scattering neglected. For a reference, the band radiances in 16 streams (eight angles for both upward and downward radiation) are calculated using DISORT with the cloud scattering fully considered using a 16-term Legendre expansion.

\section{a. Ice cloud optical property parameterizations}

Ice cloud scattering properties depend on the particle shape and size distribution, which vary temporally and spatially (Baran 2005, 2012). For our simulations, ice particle shapes are fixed and the size distribution is parameterized with a single parameter, that being the effective radius $\left(R_{e}\right)$. It is proportional to the ratio of the total volume to the total projected area for the particles in the habit/size distribution (Hansen and Travis 1974; Foot 1988), whereas the size distribution width (effective variance) is fixed. We implement two ice cloud optical property parameterizations in addition to the spherical and rough column parameterizations built into RRTM as mentioned previously. For each ice model, the optical properties are calculated for a number of wavelengths within each band before being averaged; the band-averaged optical properties are used for the calculations. A Planck function with a temperature of $250 \mathrm{~K}$ (about the temperature in the middle of the

TABLE 2. Atmospheric and cloud profile inputs for the 2160 RRTM simulations.

\begin{tabular}{|c|c|}
\hline Four ice particle models & $\begin{array}{l}\text { Spherical model } \\
\text { Rough column model } \\
\text { Aggregate model } \\
\text { THM }\end{array}$ \\
\hline Six atmospheric profiles & $\begin{array}{l}\text { U.S. Standard Atmosphere, } 1976 \\
\text { Tropical } \\
\text { Midlatitude summer } \\
\text { Midlatitude winter } \\
\text { High-latitude summer } \\
\text { High-latitude winter }\end{array}$ \\
\hline $\begin{array}{l}\text { Three single-layer ice cloud } \\
\text { altitudes }\left(H_{\mathrm{cld}}\right)\end{array}$ & 5,8, and $11 \mathrm{~km}$ \\
\hline Five ice particle effective radii & $10,20,30,40$, and $50 \mu \mathrm{m}$ \\
\hline $\begin{array}{l}\text { Six ice cloud optical thicknesses } \\
\text { (in geometric limit) }\end{array}$ & $0.1,0.3,1.0,2.0,5.0$, and 10.0 \\
\hline
\end{tabular}



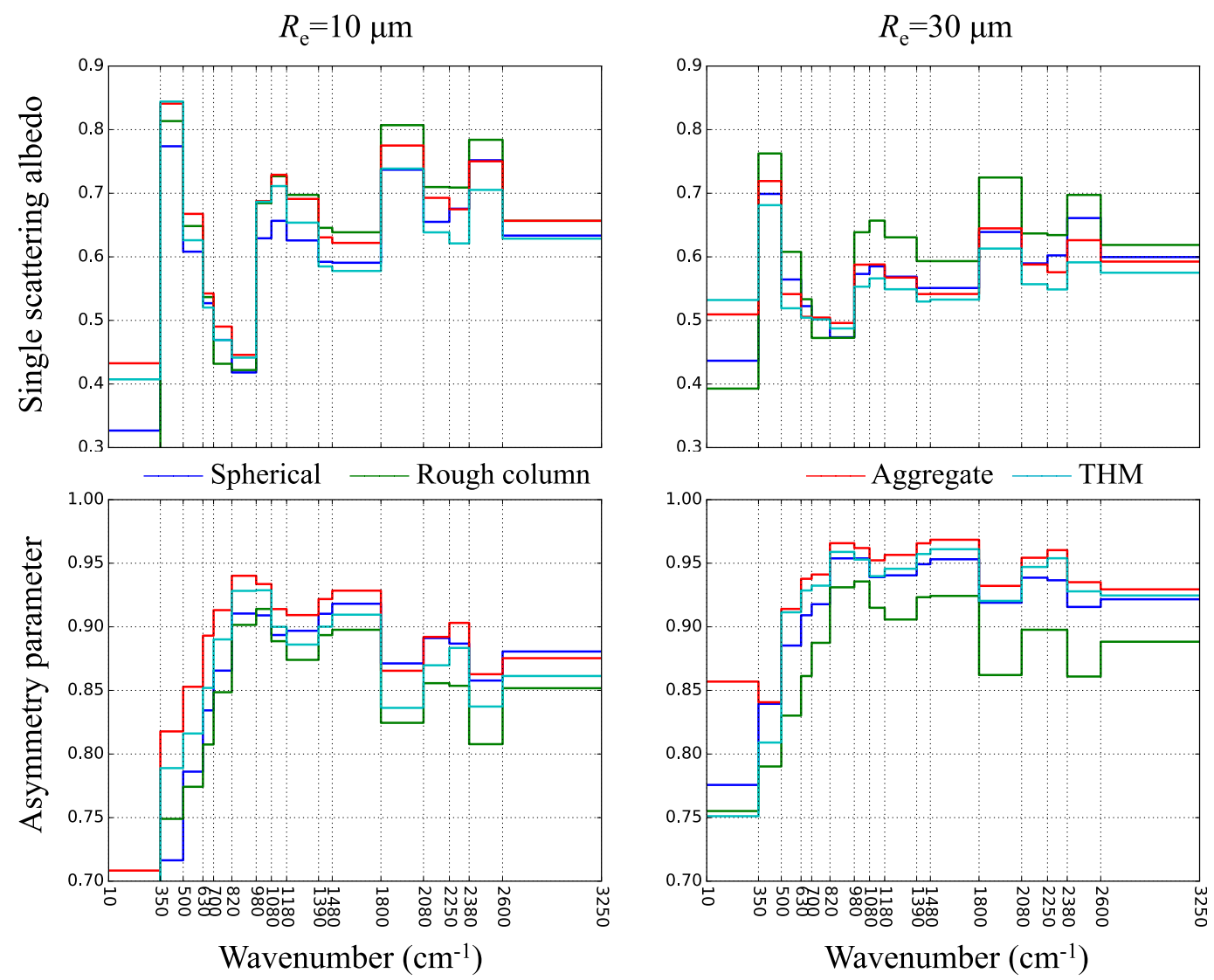

FIG. 1. Scattering property parameterizations of four ice particle models for RRTM, which divides the longwave spectral region (10-3250 $\left.\mathrm{cm}^{-1}\right)$ into 16 bands for radiation calculations (vertical dashed lines). Columns are for $R_{e}=$ (left) 10 and (right) $30 \mu \mathrm{m}$. Rows are (top) single-scattering albedo and (bottom) asymmetry factor.

troposphere) is used as the weighting function for averaging. The size distribution is a gamma function with an effective variance fixed to be 0.1 , consistent with that used in the MODIS Collection 6 and CERES-MODIS retrieval products.

Figure 1 shows the scattering property parameterizations of four ice particle models for two effective radii, 10 and $30 \mu \mathrm{m}$, as functions of the RRTM bands. Note that smaller particles have larger $\omega$ and smaller $g$, both of which lead to stronger scattering effects. For the atmospheric window bands (about $700-1200 \mathrm{~cm}^{-1}$ ), $\omega$ is about $0.4-0.7$ and $g$ is about 0.9 . This means ice clouds scatter nearly as much thermal radiation as they absorb. Because the gas absorption in the window bands is weak, ice cloud scattering plays a more important role in the radiative transfer than in other bands. The difference between the four ice models is roughly 0.1 for $\omega$ and 0.03 for $g$. In particular, the rough column model tends to have the largest $\omega$ and smallest $g$ and thus has the strongest scattering effect. The aggregate model has moderate $\omega$ and the largest $g$. Natural ice cloud particles have even more complicated geometries than the four ice models presented (Baran 2009). However, we use the four ice particle models to represent the natural variability of particle geometries and to test the performance of the newly developed schemes without losing too much generality.

\section{b. RRTM simulations}

We implement the four schemes discussed in section 2 into the standard RRTM release (version 3.3): the Chou scaling approach, the similarity principle scaling approach, the Chou scaling-adjustment scheme, and the similarity scaling-adjustment scheme. In addition, there are two schemes available in RRTM: one neglects scattering and the other uses DISORT to more accurately solve the radiative transfer equation.

For each of the 2160 atmosphere-cloud profiles, we performed irradiance calculations with these six schemes. The DISORT calculations are performed with 16 streams and a 16-order Legendre expansion, the results of which serve as a reference for the error estimation of the other five schemes. The DISORT calculation was compared to the rigorous line-by-line DISORT 

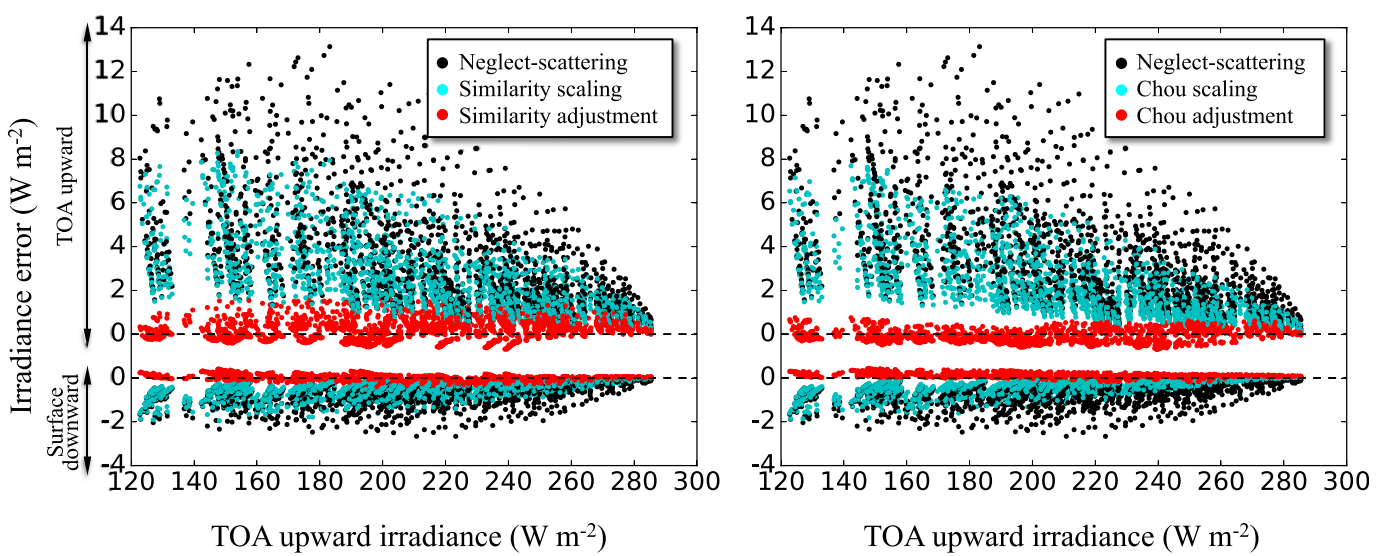

FIG. 2. Error of TOA upward and surface downward irradiances vs reference TOA upward irradiance scatterplot of five schemes: neglect scattering (black), (left) similarity scaling (cyan) and similarity adjustment (red), and (right) Chou scaling (cyan), and Chou adjustment (red). An RRTM calculation with scattering fully considered using 16-stream DISORT serves as the reference. Scattering is not explicitly considered in the five schemes; therefore, they consume similar computational time. For each scheme, radiances are calculated at three angles independently and integrated to obtain irradiance. Irradiances calculated using two to four angles (two and four angle solutions are not shown) are very similar, except when using one angle, which has large errors even for clear sky. A total of 2160 atmosphere-cloud profiles, including various atmospheric profiles, ice cloud particle shape models, cloud heights, optical thicknesses, and effective radii, are used.

(Turner et al. 2003; Turner 2005) benchmark and was within $2 \mathrm{~W} \mathrm{~m}^{-2}$ in terms of TOA irradiance. (We compared RRTM 16-stream simulations with the line-byline DISORT counterparts with a $0.01-\mathrm{cm}^{-1}$ spectral resolution for 540 of the atmosphere-cloud profiles in Table 2 with the ice particle chosen to be the aggregate model.) The surface emissivity is assumed to be unity (no reflection), which is reasonable for calculations over ocean and snow, since their emissivity is very close to 1 (about $0.97-0.99$ ) at around $11 \mu \mathrm{m}$ (Masuda et al. 1988; Li et al. 2013), the central wavelength of the thermal infrared regime, though all of the schemes are able to deal with a nonunity surface albedo by adding the reflection of the surface downward irradiance and the surface emission to obtain the surface upward irradiance. Figure 2 shows the error of five schemes in terms of TOA upward and surface downward longwave irradiances. The considerable errors in the TOA upward irradiance for the original neglectscattering scheme are noted, with an overestimation up to $14 \mathrm{~W} \mathrm{~m}^{-2}$. The two optical thickness scaling schemes reduce the maximum overestimation to about $8 \mathrm{~W} \mathrm{~m}^{-2}$. The two adjustment schemes further reduce the overestimation to less than $2 \mathrm{~W} \mathrm{~m}^{-2}$, which is in the range of the RRTM error when compared to the more rigorous line-by-line DISORT method (not shown).

The original neglect-scattering scheme underestimates the surface downward irradiance by less than $2 \mathrm{~W} \mathrm{~m}^{-2}$ because the cloud reflection caused by scattering of the upward radiation is ignored. The scaling schemes increase the cloud optical thickness and therefore increase the downward blackbody radiation and reduce the underestimation. With the adjustment schemes, the ignored cloud reflection is compensated for in the downward irradiance and therefore the underestimation of surface downward irradiance is further reduced to less than $0.5 \mathrm{~W} \mathrm{~m}^{-2}$. Both of the adjustment schemes have similar performance in reducing the surface downward and TOA upward irradiance errors. The irradiances shown in Fig. 2 are obtained by integrating radiances calculated for three angles in the upward and downward directions. We observe that the results obtained using two, three, and four angles are nearly the same. However, use of only a single angle results in significant errors as shown in Fig. 3 even though the scaling and adjustment schemes have reduced the error. This significant error could be due to the quadrature selection. RRTM uses the Gaussian quadrature for the radiance calculation and integration [for a single angle the zenith angle is $\left.\cos ^{-1}\left(1 / 3^{1 / 2}\right)\right]$, which has been suggested to be less accurate than an empirical choice of $\cos ^{-1}(1 / 1.66)$ (Fu et al. 1997). This issue is solved in the RRTMG by optimizing the angle for a few different bands for a range of total water column values.

To demonstrate the performance of the new adjustment schemes, Fig. 4 shows the comparison of errors between the Chou scaling-adjustment scheme and a four-stream approximation (listed in Table 2), which explicitly considers the cloud scattering. The two schemes have similar accuracies with errors smaller than about 


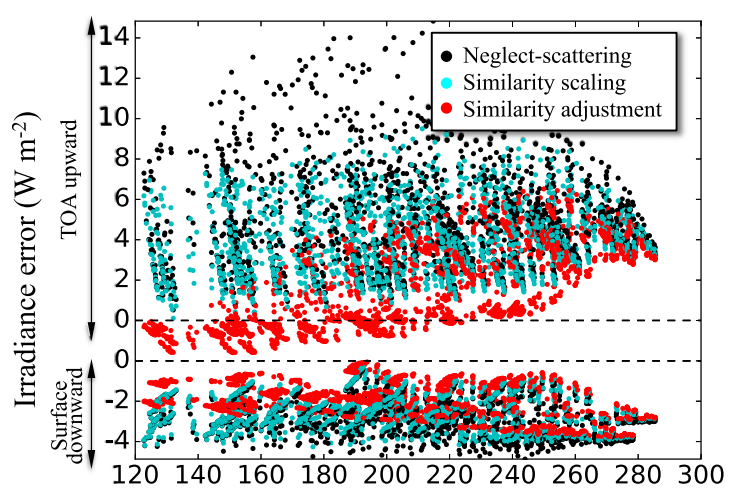

TOA upward irradiance $\left(\mathrm{W} \mathrm{m}^{-2}\right)$

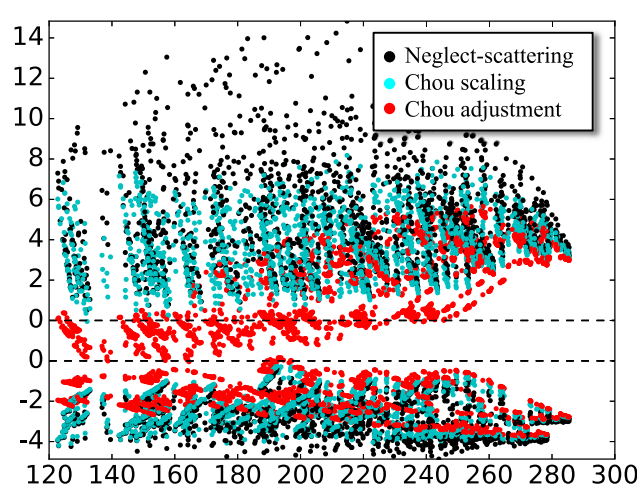

TOA upward irradiance $\left(\mathrm{W} \mathrm{m}^{-2}\right)$

FIG. 3. As in Fig. 2, but with the radiance calculated for one zenith angle for both upward and downward radiation.

$1 \mathrm{~W} \mathrm{~m}^{-2}$ in terms of TOA upward irradiance, but the four-stream approximation is demonstrably worse for optically thin clouds. The four-stream approximation consumes a computational time of about 20 times that of the adjustment scheme. Therefore, the adjustment schemes have both better accuracy and computational efficiency than the four-stream approximation.

Figure 5 shows the TOA upward irradiance error as a function of visible optical thickness for different values of the cloud height and cloud effective radius. The original neglect-scattering scheme always overestimates the TOA upward irradiance and is at a maximum for intermediate optical thickness $(\sim 1)$, which is consistent with Kuo et al. (2017). Qualitatively, the scattering is less effective for optically thin clouds, while for optically thick clouds the effect of scattering is partly offset by the absorption. The overestimation decreases with $R_{e}$ because the impact of scattering decreases with $R_{e}$ as a result of $\omega$ increasing and $g$ decreasing with $R_{e}$ in the atmospheric window spectral region. The overestimation increases with cloud height because the upward irradiance resulting from scattering in a higher cloud layer is less affected by the atmosphere above the cloud. The two scaling schemes reduce the overestimation significantly for small and intermediate optical thicknesses but approach the neglect-scattering scheme for large optical thicknesses. In fact, the no-scattering simulations always approach a constant value for large optical thickness. The two adjustment schemes further reduce the overestimation to less than $2 \mathrm{~W} \mathrm{~m}^{-2}$ over the entire range of optical thicknesses.

Figure 6 is similar to Fig. 5, but it shows the error of surface downward irradiance as a function of visible optical thickness. The original neglect-scattering scheme underestimates the surface downward irradiance and similarly has a maximum value for intermediate optical thickness. The error also decreases with $R_{e}$ but decreases with height, because the cloud reflection of upward radiation is less affected by the atmosphere beneath the clouds. The two scaling schemes again reduce the error significantly for thin and intermediate optical thicknesses but approach the results from the neglect-scattering scheme for high optical thicknesses. Both of the adjustment schemes reduce the errors for all optical thickness values to less than $0.5 \mathrm{~W} \mathrm{~m}^{-2}$.

Figure 7 shows the vertical distribution of the heating rate error for each of the five schemes for an intermediate $(\sim 1)$ cloud optical thickness. The original neglect-scattering scheme basically underestimates the heating rate beneath cloud by about $0.1 \mathrm{~K} \mathrm{day}^{-1}$, indicating a cooling effect on the atmosphere, consistent with the overestimation of the TOA upward irradiance. The cloud scattering plays a role in reflecting upward thermal radiance and warming the atmosphere below the cloud. The two

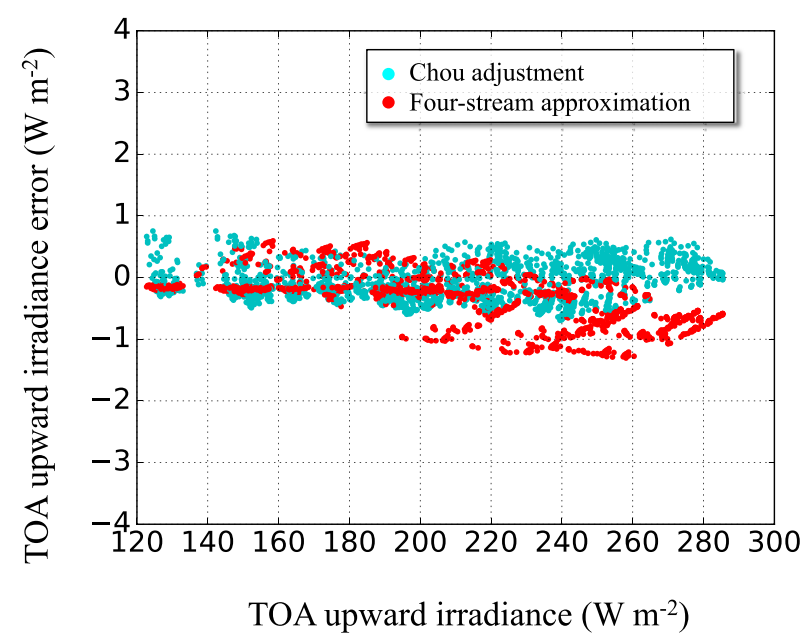

FIG. 4. Comparison of TOA upward irradiance error between the adjustment scheme based on Chou's scaling scheme (cyan) and a four-stream approximation (red). 


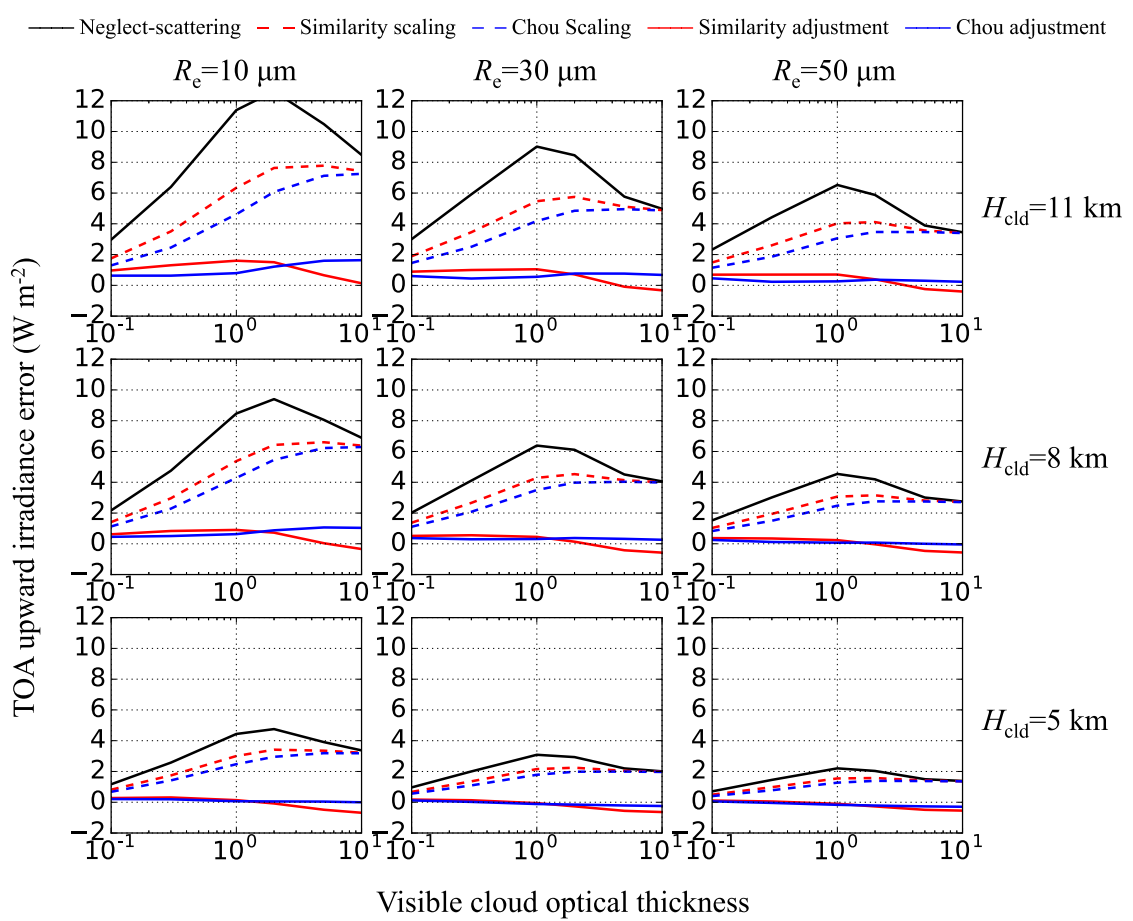

FIG. 5. TOA longwave irradiance errors of five schemes as functions of visible optical thickness: neglect scattering (solid black), similarity scaling (dashed red), Chou scaling (dashed blue), similarity adjustment (solid red), and Chou adjustment (solid blue). Columns are for different effective radii of (left) 10, (middle) 30, and (right) $50 \mu \mathrm{m}$. Rows are for different cloud heights at (top) 11 , (middle) 8 , and (bottom) $5 \mathrm{~km}$. Radiances at three angles are calculated and integrated to obtain irradiances. The rough column ice model and the U.S. Standard Atmosphere, 1976, profile are used.

scaling schemes reduce this heating rate error to less than $0.01 \mathrm{~K} \mathrm{day}^{-1}$, whereas the two adjustment schemes further reduce it to almost zero. The vertical average heating rate error is reduced by the two scaling schemes and further reduced by the two adjustment schemes. An accurate calculation of the thermal heating rate is especially important at high latitudes, where the thermal radiation is comparable to, or may even dominate, the contribution from solar radiation.

The ice cloud parameterization also leads to great uncertainties in the radiative transfer simulations. It is uncertain whether the longwave scattering effect is negligible compared to the effect of ice cloud parameterization. To gain further insight, we investigate the effect of ice cloud parameterization on the thermal radiative transfer. Figure 8 shows the TOA longwave radiation as a function of visible optical thickness for the four different ice cloud parameterizations. For the same $R_{e}$ and visible optical thickness, different ice cloud parameterizations have the same ice water path. The rough column model always results in the smallest TOA thermal loss, leading to a warming effect in contrast with the other three models. The aggregate model and THM result in the largest values of the TOA thermal radiation and are nearly the same with a difference of less than $2 \mathrm{~W} \mathrm{~m}^{-2}$. The differences between four ice models are larger for intermediate optical thickness values, at most $20 \mathrm{~W} \mathrm{~m}^{-2}$. The differences are larger for high clouds and larger cloud effective radii because the optical properties of larger particles are more sensitive to particle shape. The sensitivity of TOA irradiance to $R_{e}$ is relatively weak compared with the parameterizations, especially for the aggregate model and THM. Thus, in GCMs, it seems reasonable to assume a constant value of $R_{e}$ for longwave calculations. However, for the rough column model, the TOA irradiance for an $R_{e}$ of $10 \mu \mathrm{m}$ is still larger than that for an $R_{e}$ of $30 \mu \mathrm{m}$ by up to about $15 \mathrm{~W} \mathrm{~m}^{-2}$, indicating a potential cooling effect when the cloud particle size is reduced, for example, because of aerosol pollution.

\section{Discussion and conclusions}

This study demonstrates that neglecting cloud longwave scattering can result in a significant overestimation of the top-of-the-atmosphere (TOA) thermal radiation, 


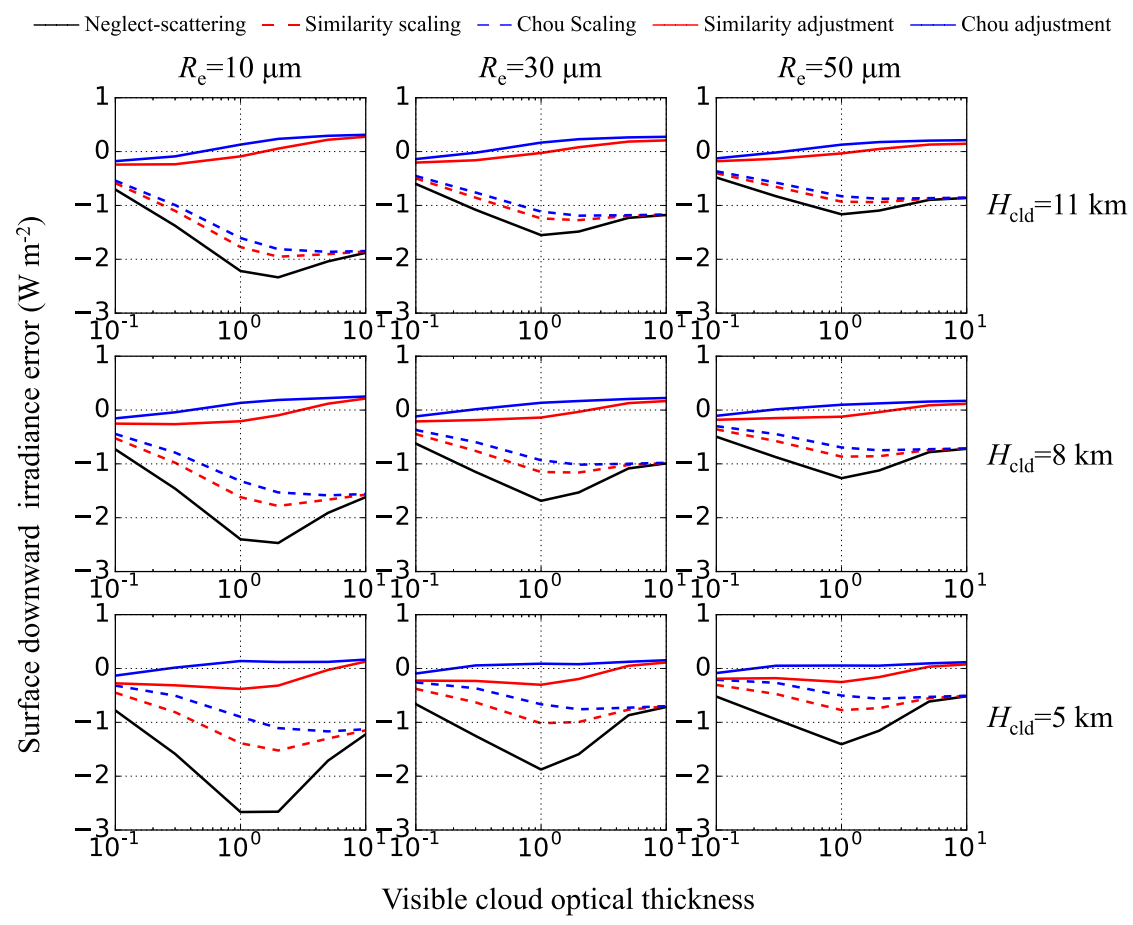

FIG. 6. As in Fig. 5, but for the surface downward irradiance error.

which in turn leads to a spurious cooling effect below the clouds. The overestimation can be as large as $14 \mathrm{~W} \mathrm{~m}^{-2}$ depending on the cloud thickness, altitude, and optical properties. In general, the overestimation reaches its highest values for a higher cloud of an intermediate optical thickness with relatively small cloud particles. Additionally, neglecting cloud longwave scattering leads to an underestimate in the surface downward

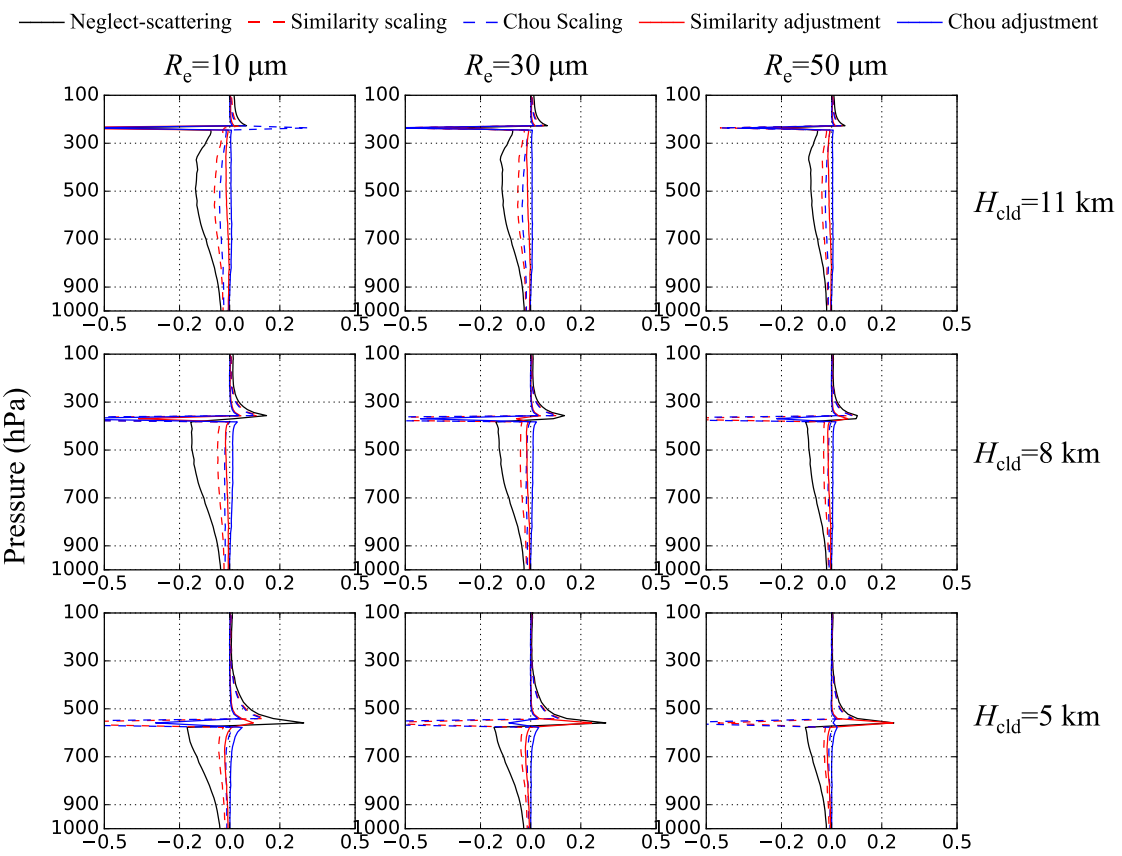

Heating rate error $\left(\mathrm{K}_{\text {day }}{ }^{-1}\right)$

FIG. 7. As in Fig. 5, but for heating rate error of the five schemes as a function of height. The cloud optical thickness is 1 . 


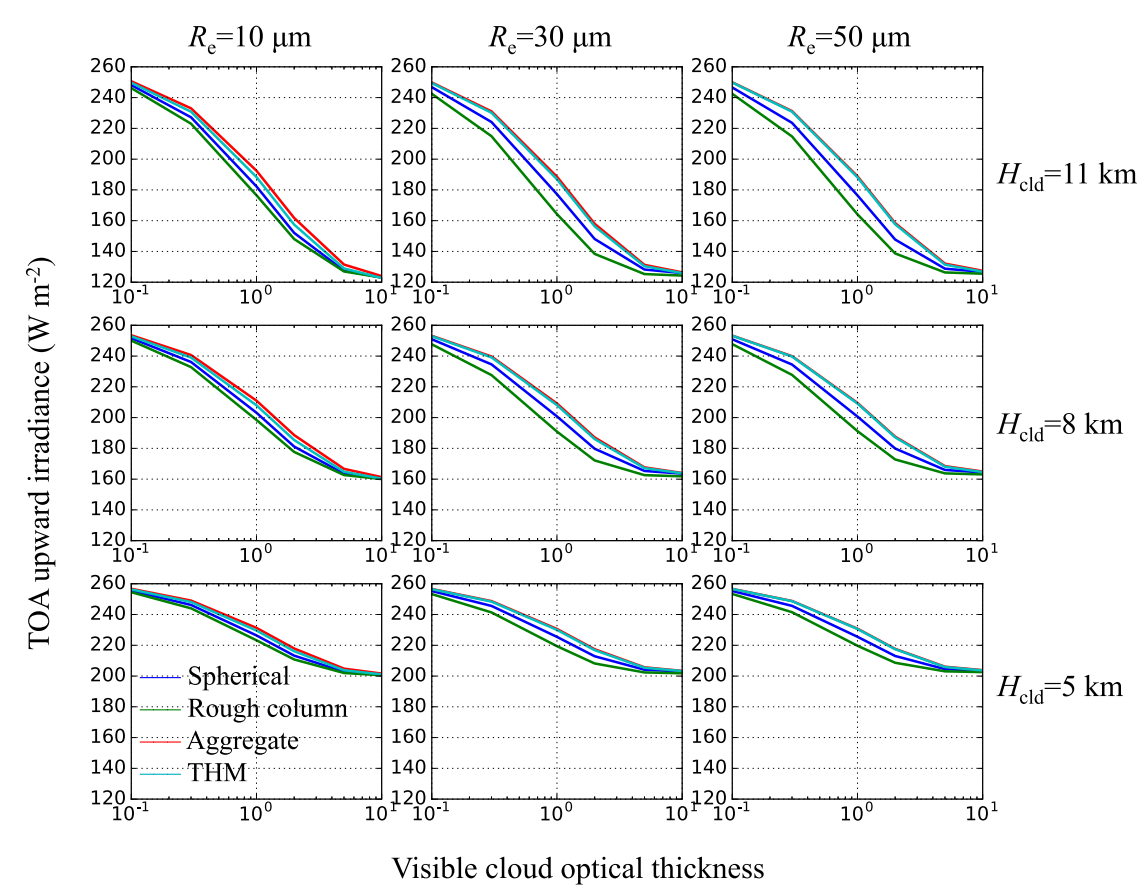

FIG. 8. As in Fig. 5, but for TOA longwave irradiance as a function of visible cloud optical thickness for four different ice cloud models: spherical (dark blue), rough column (green), aggregate (red), and THM (light blue). RRTM with 16-stream DISORT is used in the simulations.

irradiance-or in other words, it overestimates the surface net upward irradiance by up to $2 \mathrm{~W} \mathrm{~m}^{-2}$. Neglecting multiple scattering in lower clouds results in larger errors in the surface downward irradiance.

Theoretically, clouds scatter longwave upward radiation to the side and backward directions and therefore reduce the thermal radiation reaching the TOA while also increasing the thermal radiation reflected back to surface. Thus, neglecting scattering overestimates TOA thermal radiation and underestimates surface downward radiation. By increasing the cloud absorptive optical thickness while still neglecting scattering, less thermal radiation reaches the TOA and more blackbody radiation emanating from clouds reaches the surface, and thus both TOA and surface radiation errors are reduced. However, for optically thick clouds, increasing the cloud absorptive optical thickness offers little improvement because the irradiance changes little with increasing optical thickness. We applied the similarity principle and Chou et al.'s (1999) scaling schemes to RRTM and verified the conclusion stated above. For optically thick clouds, the TOA irradiance overestimation can still reach $8 \mathrm{~W} \mathrm{~m}^{-2}$.

We developed adjustment schemes for both the similarity principle and Chou et al. (1999) scaling schemes, in which the downward radiances are calculated and then used to adjust the upward radiances. In an iterative process, the upward radiances are again used to adjust the downward radiances. A negative adjustment term for the upward radiance is derived that increases with cloud optical thickness and approaches a constant for very thick clouds. The adjustment term for the downward radiance is similar but positive. In such a way, the TOA and surface irradiance errors are further reduced. We applied these two schemes to RRTM and found that the TOA upward and surface downward irradiance errors are reduced to less than 2 and $0.5 \mathrm{~W} \mathrm{~m}^{-2}$, respectively, over a wide range of optical thickness values. The comparison with the four-stream approximation shows that the new adjustment schemes are more accurate than the four-stream approximation and about 20 times faster computationally.

The simulations are performed for various atmospheric profiles, cloud heights, optical thicknesses, ice particle sizes, and cloud optical property parameterizations. As a reference to evaluate the error, the cloud scattering is considered with a 16-stream DISORT computation in RRTM, which has been shown to agree with rigorous line-by-line DISORT benchmarks within $2 \mathrm{~W} \mathrm{~m}^{-2}$ for all sky conditions.

In addition, considering that the similarity principle failed to improve the two-stream approximation for multiple layers combined with the adding method, we further 
verified the new schemes for multiple cloud layers and obtained very similar errors as for single layers (not shown). We conclude that the adjustment schemes are effective in improving the accuracy of longwave radiative transfer for virtually all sky conditions.

In the RRTM simulations, we found that the radiance computed at a single Gaussian quadrature angle is insufficient for the irradiance integral, similar to the study by $\mathrm{Fu}$ et al. (1997). More than one Gaussian quadrature angle results in relatively accurate irradiance integrals. Thus, we suggest using at least two Gaussian quadrature angles to solve the longwave radiative transfer equation in a broadband radiative transfer model such as RRTM.

Cloud longwave scattering has a warming effect beneath clouds of up to $0.1 \mathrm{~K} \mathrm{day}^{-1}$, but this is neglected in most GCMs. The scaling and adjustment schemes help to account for such a warming effect.

We also found that the broadband irradiances are sensitive to the ice cloud optical property parameterization. The differences between different parameterizations are of similar magnitude to the scattering effect itself. As studies involving the remote sensing of clouds are leading to more accurate cloud microphysics parameterizations, the new schemes, in conjunction with more realistic cloud microphysical illustrations, will help improve the energy budget estimation.

Finally, we note that RRTMG, as a GCM version of RRTM, uses one angle following Fu et al. (1997) with optimizations in a few bands as a function of the total column water. Further improvement of the RRTMG by using more than one angle and the adjustment schemes will be explored in future work.

Acknowledgments. This study was supported by the National Science Foundation (AGS-1632209) and partly by the endowment funds related to the David Bullock Harris Chair in Geosciences at the College of Geosciences, Texas A\&M University. The effort of coauthor Huang is supported by the U.S. Department of Energy, Office of Science, Office of Biological and Environmental Research, Climate and Environmental Science Division under Award DE-SC0012969 to the University of Michigan. The simulations are conducted through the Texas A\&M supercomputing facility.

\section{APPENDIX}

\section{Physical Meaning of the Similarity Scaling and Adjustment}

To understand the physical meaning of the similarity principle and the adjustment term, we simplify the atmosphere to be a single cloudy layer with a uniform temperature of $T_{c}$, an optical thickness of $\tau_{c}$, a singlescattering albedo of $\omega$, and an asymmetry factor of $g$. We further assume that the radiation is one-dimensional, meaning that we can think of this as a representation of upward and downward irradiances, so then we can use an exact solution of the irradiance (Kattawar and Plass 1973). The surface temperature and emissivity are assumed to be $T_{S}$ and unity, respectively. This simplified model approximately describes the behavior of the radiative transfer in the atmosphere and is used to understand the similarity principle and the adjustment term. We consider the single-scattering scenario, where $\tau_{c} \ll 1$. In this case, the cloud layer absorbs a fraction of $(1-\omega)$ of the total extinction of the upward irradiance from the surface, and scatters a fraction of $\omega[(1+g) / 2]$ upward and a fraction of $\omega[(1-g) / 2]$ back downward to the surface. Then the TOA upward irradiance is

$$
\begin{aligned}
F^{\uparrow}(0)= & e^{-\tau_{c}} \pi B_{S}+\left(1-e^{-\tau_{c}}\right) \omega \frac{1+g}{2} \pi B_{S} \\
& +\left(1-e^{-\tau_{c}}\right)(1-\omega) \pi B_{c},
\end{aligned}
$$

where $F^{\uparrow}$ is the upward irradiance as a function of $\tau$, with $F^{\uparrow}(0)$ being the TOA irradiance. The quantities $B_{S}$ and $B_{c}$ are the blackbody radiance for temperatures $T_{S}$ and $T_{c}$, respectively. The quantities $\pi B_{S}$ and $\pi B_{c}$ are thus the blackbody irradiances of the surface and the cloud layer, respectively. The three terms on the right-hand side of Eq. (A1) are the transmitted surface irradiance to the TOA, scattered surface irradiance at the TOA, and atmospheric thermal irradiance at the TOA, respectively. For an optically thin layer $\left(\tau_{c} \ll 1\right), e^{-\tau_{c}} \approx 1-\tau_{c}$, and Eq. (A1) is simplified as

$$
\begin{aligned}
F^{\uparrow}(0) & =\left(1-\tau_{c}\right) \pi B_{S}+\tau_{c} \omega \frac{1+g}{2} \pi B_{S}+\tau_{c}(1-\omega) \pi B_{c} \\
& =\left[1-\left(1-\omega \frac{1+g}{2}\right) \tau_{c}\right] \pi B_{S}+(1-\omega) \tau_{c} \pi B_{c} .
\end{aligned}
$$

Now we consider neglecting the scattering by replacing $\tau_{c}$ and $\omega$ with $\tau_{c}(1-\omega)$ and zero, respectively, and removing the scattering term in Eq. (A2). Then the solution is

$$
\begin{aligned}
F^{\uparrow}(0) & =\left[1-(1-\omega) \tau_{c}\right] \pi B_{S}+0+\tau_{c}(1-\omega) \pi B_{c} \\
& =\left[1-(1-\omega) \tau_{c}\right] \pi B_{S}+(1-\omega) \tau_{c} \pi B_{c} .
\end{aligned}
$$

Simply setting $g$ to be 1 in Eq. (A2) also results in Eq. (A3). From the exact solution [Eq. (A2)] to the neglectscattering solution Eq. (A3), the transmitted surface blackbody radiative irradiance to the TOA increases 
by $\omega \tau_{c} \pi B_{S}$ because the optical thickness decreases; the upward scattered irradiance decreases by $\tau_{c} \omega[(1+g) / 2] \pi B_{S}$ to zero because the scattering is neglected; and the atmospheric emission to TOA remains unchanged because the absorptive optical thickness does not change. In sum, the neglect-scattering solution is $\tau_{c} \omega[(1-g) / 2] \pi B_{c}$ larger than the exact solution.

Further, we consider the similarity principle scaling solution by replacing $\tau_{c}$ and $\omega$ with $\tau_{c}[1-\omega(1+g) / 2]$
TABLE A1. Error of the schemes in terms of TOA upward irradiance for the simplified single-atmospheric-layer model.

\begin{tabular}{lc}
\hline \hline \multicolumn{1}{c}{ Scheme } & Error \\
\hline Neglect scattering & $\tau_{c} \omega(1-g) / 2 \times \pi B_{S}$ \\
Similarity principle scaling & $\tau_{c} \omega(1-g) / 2 \times \pi B_{c}$ \\
Similarity scaling adjustment & 0 \\
\hline
\end{tabular}

and zero, respectively, and removing the scattering term in Eq. (A2). Then the solution is

$$
F^{\uparrow}(0)=\left[1-\left(1-\omega \frac{1+g}{2}\right) \tau_{c}\right] \pi B_{S}+0+\tau_{c}\left(1-\omega \frac{1+g}{2}\right) \pi B_{c}=\left[1-\left(1-\omega \frac{1+g}{2}\right) \tau_{c}\right] \pi B_{S}+\left(1-\omega \frac{1+g}{2}\right) \tau_{c} \pi B_{c} .
$$

From the exact solution [Eq. (A2)] to the similarity principle scaling solution [Eq. (A4)], the transmitted surface blackbody irradiance to TOA increases by $\omega[(1+g) / 2] \tau_{c} \pi B_{S}$; the upward scattered irradiance decreases by $\tau_{c} \omega[(1+g) / 2] \pi B_{S}$ to zero to offset the transmission increase; and the atmospheric emission to TOA increases by $\tau_{c} \omega[(1-g) / 2] \pi B_{c}$ Taken together, the similarity principle scaling solution is $\tau_{c} \omega[(1-g) / 2] \pi B_{c}$ larger than the exact solution.

The error of the similarity principle scaling solution $\tau_{c} \omega[(1-g) / 2] \pi B_{c}$ is basically smaller than that of the neglect-scattering solution $\tau_{c} \omega[(1-g) / 2] \pi B_{S}$ because the cloud temperature is lower than the surface temperature, especially when the cloud layer is high. The error of the similarity scaling scheme comes from the increase of the cloud thermal radiation resulting from larger absorptive optical thickness.

Moreover, we consider an adjustment term applied to the similarity principle scaling solution, which according to Eq. (21) is [replacing $(1-b)$ with $(1+g) / 2]$

$$
\begin{aligned}
F^{\uparrow s s}(0) & =\frac{\omega \frac{1-g}{2}}{2\left(1-\omega \frac{1+g}{2}\right)}\left\{\left[F^{\downarrow}(0)-\pi B_{c}\right]-\left[F^{\downarrow}\left(\tau_{c}\right)-\pi B_{c}\right] \exp \left[-\left(1-\omega \frac{1+g}{2}\right) \tau_{c}\right]\right\} \\
& =\frac{\omega \frac{1-g}{2}}{2\left(1-\omega \frac{1+g}{2}\right)}\left\{-\pi B_{c}-\left(1-\omega \frac{1+g}{2}\right) \tau_{c} \pi B_{c}+\pi B_{c}\left[1-\left(1-\omega \frac{1+g}{2}\right) \tau_{c}\right]\right\}=-\omega \frac{1-g}{2} \tau_{c} \pi B_{c},
\end{aligned}
$$

where $F^{\uparrow s s}$ is the specific solution of the upward irradiance and also is a function of $\tau$. Note that the transmissivity of the cloud layer is $\exp \left\{-[1-\omega(1+\mathrm{g}) / 2] \tau_{c}\right\}$ and the relation

$$
\begin{aligned}
F^{\downarrow}(0) & =0 \\
F^{\downarrow}\left(\tau_{c}\right) & =\left(1-\omega \frac{1+g}{2}\right) \tau_{c} \pi B_{c}
\end{aligned}
$$

is used in the derivation of Eq. (A5). The errors of the three schemes given above are listed in Table A1. It is useful to note that the adjustment term Eq. (A5) offsets completely the error of the similarity principle scaling solution [Eq. (A4)], meaning that the similarity scalingadjustment solution is the same as the exact solution in this simplified case. The physical meaning of this adjustment term is to offset the overestimated upward cloud thermal radiation caused by the scaling for optically thin clouds. For optically thick clouds, the scaling scheme no longer has an effect on the TOA irradiance, which equals the cloud blackbody radiation, and is larger than the true value because the cloud emissivity is less than unity. The physical meaning of the adjustment term is to reduce this overestimation resulting from nonunity emissivity. 


\section{REFERENCES}

Baran, A. J., 2005: The dependence of cirrus infrared radiative properties on ice crystal geometry and shape of the size distribution function. Quart. J. Roy. Meteor. Soc., 131, 1129-1142, https://doi.org/10.1256/qj.04.91.

_ 2009: A review of the light scattering properties of cirrus. J. Quant. Spectrosc. Radiat. Transfer, 110, 1239-1260, https:// doi.org/10.1016/j.jqsrt.2009.02.026.

_ 2012: From the single-scattering properties of ice crystals to climate prediction: A way forward. Atmos. Res., 112, 45-69, https://doi.org/10.1016/j.atmosres.2012.04.010.

Chou, M.-D., K.-T. Lee, S.-C. Tsay, and Q. Fu, 1999: Parameterization for cloud longwave scattering for use in atmospheric models. J. Climate, 12, 159-169, https://doi.org/ 10.1175/1520-0442-12.1.159.

COESA, 1976: U.S. Standard Atmosphere, 1976. NOAA, 227 pp.

Costa, S. M. S., and K. P. Shine, 2006: An estimate of the global impact of multiple scattering by clouds on outgoing long-wave radiation. Quart. J. Roy. Meteor. Soc., 132, 885-895, https:// doi.org/10.1256/qj.05.169.

Ding, J., P. Yang, G. W. Kattawar, M. D. King, S. Platnick, and K. G. Meyer, 2017: Validation of quasi-invariant ice cloud radiative quantities with MODIS satellite-based cloud property retrievals. J. Quant. Spectrosc. Radiat. Transfer, 194, 47-57, https://doi.org/10.1016/j.jqsrt.2017.03.025.

Edmonds, A. R., 2016: Angular Momentum in Quantum Mechanics. Princeton University Press, $160 \mathrm{pp}$.

Foot, J. S., 1988: Some observations of the optical properties of clouds. II: Cirrus. Quart. J. Roy. Meteor. Soc., 114, 145-164, https://doi.org/10.1002/qj.49711447908.

Fu, Q., K. N. Liou, M. C. Cribb, T. P. Charlock, and A. Grossman, 1997: Multiple scattering parameterization in thermal infrared radiative transfer. J. Atmos. Sci., 54, 2799-2812, https://doi. org/10.1175/1520-0469(1997)054<2799:MSPITI>2.0.CO;2.

_- P. Yang, and W. B. Sun, 1998: An accurate parameterization of the infrared radiative properties of cirrus clouds for climate models. J. Climate, 11, 2223-2237, https://doi.org/10.1175/ 1520-0442(1998)011<2223:AAPOTI>2.0.CO;2.

Hansen, J. E., 1969: Absorption-line formation in a scattering planetary atmosphere: A test of van de Hulst's similarity relations. Astrophys. J., 158, 337-349, https://doi.org/10.1086/150196.

— spheres. Space Sci. Rev., 16, 527-610, https://doi.org/10.1007/ BF00168069.

, G. Russell, D. Rind, P. Stone, A. Lacis, S. Lebedeff, R. Ruedy, and L. Travis, 1983: Efficient three-dimensional global models for climate studies: Models I and II. Mon. Wea. Rev., 111, 609-662, https://doi.org/10.1175/1520-0493(1983) $111<0609$ :ETDGMF $>2.0 . \mathrm{CO} ; 2$.

$\mathrm{Hu}, \mathrm{Y}$. X., and K. Stamnes, 1993: An accurate parameterization of the radiative properties of water clouds suitable for use in climate models. J. Climate, 6, 728-742, https://doi.org/10.1175/ 1520-0442(1993)006<0728:AAPOTR > 2.0.CO;2.

Iacono, M. J., E. J. Mlawer, S. A. Clough, and J.-J. Morcrette, 2000: Impact of an improved longwave radiation model, RRTM, on the energy budget and thermodynamic properties of the NCAR community climate model, CCM3. J. Geophys. Res., 105, 14 873-14 890, https://doi.org/10.1029/2000JD900091.

Joseph, E., and Q. Min, 2003: Assessment of multiple scattering and horizontal inhomogeneity in IR radiative transfer calculations of observed thin cirrus clouds. J. Geophys. Res., 108, 4380, https://doi.org/10.1029/2002JD002831.
Joseph, J. H., W. J. Wiscombe, and J. A. Weinman, 1976: The deltaEddington approximation for radiative flux transfer. J. Atmos. Sci., 33, 2452-2459, https://doi.org/10.1175/1520-0469(1976) $033<2452$ :TDEAFR $>2.0$. CO 2 .

Kattawar, G. W., and G. N. Plass, 1973: Interior radiances in optically deep absorbing media-I. Exact solutions for onedimensional model. J. Quant. Spectrosc. Radiat. Transfer, $\mathbf{1 3}$ 1065-1080, https://doi.org/10.1016/0022-4073(73)90080-0.

Kay, J. E., and Coauthors, 2015: The Community Earth System Model (CESM) large ensemble project: A community resource for studying climate change in the presence of internal climate variability. Bull. Amer. Meteor. Soc., 96, 1333-1349, https://doi.org/10.1175/BAMS-D-13-00255.1.

Key, J., 1996: Streamer user's guide. Boston University Tech. Rep. 96-01, 85 pp.

King, M. D., 1981: A method for determining the single scattering albedo of clouds through observation of the internal scattered radiation field. J. Atmos. Sci., 38, 2031-2044, https://doi.org/ 10.1175/1520-0469(1981)038<2031:AMFDTS>2.0.CO;2.

Kuo, C.-P., P. Yang, X. Huang, D. Feldman, M. Flanner, C. Kuo, and E. J. Mlawer, 2017: Impact of multiple scattering on longwave radiative transfer involving clouds. J. Adv. Model. Earth Syst., 9, 3082-3098, https://doi.org/10.1002/2017MS001117.

Li, Z.-L., H. Wu, N. Wang, S. Qiu, J. A. Sobrino, Z. Wan, B.-H. Tang, and G. Yan, 2013: Land surface emissivity retrieval from satellite data. Int. J. Remote Sens., 34, 3084-3127, https://doi.org/10.1080/01431161.2012.716540.

Liou, K. N., 1980: An Introduction to Atmospheric Radiation. Vol. 26. Academic Press, 391 pp.

Loeb, N. G., and Coauthors, 2018: Impact of ice cloud microphysics on satellite cloud retrievals and broadband flux radiative transfer model calculations. J. Climate, 31, 1851-1864, https:// doi.org/10.1175/JCLI-D-17-0426.1.

Masuda, K., T. Takashima, and Y. Takayama, 1988: Emissivity of pure and sea waters for the model sea surface in the infrared window regions. Remote Sens. Environ., 24, 313-329, https:// doi.org/10.1016/0034-4257(88)90032-6.

McKellar, B. H. J., and M. A. Box, 1981: The scaling group of the radiative transfer equation. J. Atmos. Sci., 38, 1063-1068, https:// doi.org/10.1175/1520-0469(1981)038<1063:TSGOTR >2.0.CO;2.

Mlawer, E. J., S. J. Taubman, P. D. Brown, M. J. Iacono, and S. A. Clough, 1997: Radiative transfer for inhomogeneous atmospheres: RRTM, a validated correlated-k model for the longwave. J. Geophys. Res., 102, 16 663-16 682, https://doi.org/ 10.1029/97JD00237.

Platnick, S., and Coauthors, 2017: The MODIS cloud optical and microphysical products: Collection 6 updates and examples from Terra and Aqua. IEEE Trans. Geosci. Remote Sens., 55, 502-525, https://doi.org/10.1109/TGRS.2016.2610522.

Powers, J. G., and Coauthors, 2017: The Weather Research and Forecasting (WRF) Model: Overview, system efforts, and future directions. Bull. Amer. Meteor. Soc., 98, 1717-1737, https://doi.org/10.1175/BAMS-D-15-00308.1.

Ritter, B., and J.-F. Geleyn, 1992: A comprehensive radiation scheme for numerical weather prediction models with potential applications in climate simulations. Mon. Wea. Rev., 120, 303-325, https:// doi.org/10.1175/1520-0493(1992)120<0303:ACRSFN>2.0.CO;2.

Schmidt, G. A., and Coauthors, 2006: Present-day atmospheric simulations using GISS ModelE: Comparison to in situ, satellite, and reanalysis data. J. Climate, 19, 153-192, https:// doi.org/10.1175/JCLI3612.1.

Sobolev, V. V., 1975: Rasseianie sveta $v$ atmosferakh planet (Light Scattering in Planetary Atmospheres). Pergamon Press, 256 pp. 
Stephens, G. L., P. M. Gabriel, and P. T. Partain, 2001: Parameterization of atmospheric radiative transfer. Part I: Validity of simple models. J. Atmos. Sci., 58, 3391-3409, https://doi.org/ 10.1175/1520-0469(2001)058<3391:POARTP > 2.0.CO;2.

Turner, D. D., 2005: Arctic mixed-phase cloud properties from AERI lidar observations: Algorithm and results from SHEBA. J. Appl. Meteor., 44, 427-444, https://doi.org/10.1175/JAM2208.1.

, S. A. Ackerman, B. A. Baum, H. E. Revercomb, and P. Yang, 2003: Cloud phase determination using ground-based AERI observations at SHEBA. J. Appl. Meteor., 42, 701-715, https:// doi.org/10.1175/1520-0450(2003)042<0701:CPDUGA >2.0.CO;2.

Twomey, S., and C. F. Bohren, 1980: Simple approximations for calculations of absorption in clouds. J. Atmos. Sci., 37, 2086-2095, https:// doi.org/10.1175/1520-0469(1980)037<2086:SAFCOA > 2.0.CO;2 van de Hulst, H. C., 1974: The spherical albedo of a planet covered with a homogeneous cloud layer. Astron. Astrophys., 35, 209-214.

— 1980: Multiple Light Scattering: Tables, Formulas, and Applications. Vol. 2, Academic Press, 436 pp.

Wiscombe, W. J., 1977: The delta $-M$ method: Rapid yet accurate radiative flux calculations for strongly asymmetric phase functions. J. Atmos. Sci., 34, 1408-1422, https://doi.org/ 10.1175/1520-0469(1977)034<1408:TDMRYA>2.0.CO;2.

Yang, P., L. Bi, B. A. Baum, K.-N. Liou, G. W. Kattawar, M. I. Mishchenko, and B. Cole, 2013: Spectrally consistent scattering, absorption, and polarization properties of atmospheric ice crystals at wavelengths from 0.2 to $100 \mu \mathrm{m}$. J. Atmos. Sci., 70, 330-347, https://doi.org/10.1175/JAS-D-12-039.1. 